\title{
PROCESS FAULT DIAGNOSIS USING RECURSIVE MULTIVARIATE STATISTICAL PROCESS CONTROL
}

\author{
Xun Wang, Uwe Krüger ${ }^{1}$, and George W. Irwin \\ Intelligent Systems \& Control Group \\ Queen's University of Belfast \\ Belfast BT9 5AH \\ United Kingdom
}

\begin{abstract}
Over the last few years, recursive extensions to multivariate statistical process control (MSPC) techniques have gained attention for their ability in monitoring largescale, time-varying processes. Although recursive MSPC techniques have been successfully applied in detecting faulty conditions, little interest has been shown in utilising them for diagnosis purposes. This paper addresses this issue and introduces new fault diagnosis charts that rely on recursive MSPC models. The utility of these is demonstrated using an application study on a simulation of a complex chemical process. Copyright $\mathcal{O} 2005$ IFAC
\end{abstract}

Keywords: Recursive Algorithms, Statistical Process Control, Process Models, Data Reduction, Fault Diagnosis

\section{INTRODUCTION}

Modern industrial processes often present a large number of measured variables, which are frequently sampled and usually highly correlated. MSPC methods are known to be effective in detecting and diagnosing abnormal operating conditions in such circumstances (MacGregor et al., 1991; Martin and Morris, 1996; Wise and Gallagher, 1996). Two of the most commonly used MSPC methods are Principal Component Analysis (PCA) and Partial Least Squares (PLS), (Kresta et al. 1991; MacGregor and Kourti, 1995; Kourti and MacGregor, 1995).

Gallagher et al. (1997) highlighted the fact that most industrial processes are time-varying and thus require an adaptive rather than a fixed model. For the monitoring of such processes, it is required that the model can be updated to accommodate time-varying behaviour, whilst still being able to detect abnormal conditions which violate the confidence limits, which themselves may also have to vary with time (Wang et al., 2003).

${ }^{1}$ Corresponding Author, Email: uwe.kruger@ee.qub.ac.uk; Tel: +44(0)2890 974059; Fax: +44(0)2890667023
Whilst, several authors (Helland et al., 1991; Qin, 1993, 1998; Dayal and MacGregor, 1997) have introduced recursive extensions to PLS, Li et al. (2000) showed how to establish recursive PCA. When a new observation becomes available, the PCA/PLS models are updated using this new observation together with the old model rather than the old data set. The adaptation of the PCA/PLS model also leads to adjustment of the monitoring statistics and their confidence limits, as discussed by $\mathrm{Li}$ et al. (2000) and Wang et al. (2003).

If the monitoring statistics indicate abnormal process behaviour, it is important to diagnose such behaviour in order to determine the root cause of this event. This can aid an experienced process operator to respond correctly and promptly in removing, or at least reducing, the impact of the abnormal event. The identification of potential root causes typically relies on contribution charts that describe the contribution of individual variables to the statistical monitoring statistics (MacGregor et al., 1994; MacGregor and Kourti, 1995; Morud, 1996). Miller et al. (1998) suggested plotting the process variables at particular sampling intervals in form of a bar chart. Despite numerous applications of contribution charts in con- 
ventional PCA/PLS applications, little interest has been shown in developing charts that take advantage of the adaptive models generated in recursive PCA/PLS.

The contribution of this paper is the development of two new fault diagnosis charts that are designed for the application of recursive PCA/PLS. As these charts rely on the adaptation of the PCA/PLS models, they display changes in the model coefficients. Note that although only recursive PCA/PLS are considered here, the new charts are not restricted to recursive applications only. The new charts can also be integrated in alternative adaptation techniques relating to the application of moving window PCA/PLS.

Recursive PCA and PLS are briefly reviewed in the next section. This is followed by an introduction to the new fault diagnosis charts and their applications in Sections 3 and 4 respectively. The conclusions of this work are finally given in Section 5.

\section{REVIEW OF PREVIOUS WORK}

This section provides a brief review of recursive PCA and recursive PLS, which explains the notations used in the derivation of the fault diagnosis charts.

\subsection{Recursive Principal Component Analysis}

As a well-established MSPC technique, PCA has been widely used in process monitoring (Kourti and MacGregor, 1995; Wise and Gallagher, 1996). The PCA model can be calculated either directly from the data matrix that stores the recorded reference data, denoted here as $\mathbf{Z}$ in which the observations at different time instant $k$ are stored as row vectors in consecutive order, or from the corresponding correlation matrix, referred to here as $\mathbf{S}_{Z Z}$. Using the PCA model, the data matrix approximated as follows:

$$
\mathbf{Z}=\mathbf{T R}^{T}+\mathbf{E}
$$

where $\mathbf{T}$ and $\mathbf{R}$ are score and loading matrices respectively and $\mathbf{E}$ is residual matrix. Li et al. (2000) showed computationally efficient ways of recursively updating the correlation matrix. For the $(k+1)^{\text {th }}$ observation, this update procedure relies on the previous correlation matrix ${ }_{k} \mathbf{S}_{Z Z}$ and a new observation, i.e. the $(k+1)^{\text {th }}$ observation $\mathbf{z}_{k+1}$ and involves (i) the adaptation of the mean and standard deviation of the recorded process variables, (ii) the subsequent adaptation of the correlation matrix, (iii) the determination of the parameters of the $(k+1)^{\text {th }}$ PCA model, (iv) the adaptation of the confidence limits for the univariate monitoring statistics and (v) the updating of the monitoring statistics using the previously updated monitoring model.

\subsection{Recursive Partial Least Squares}

The PLS technique decomposes the predictor or input matrix $\mathbf{X}$, including $M$ predictor variables, and the response or output matrix $\mathbf{Y}$, including $N$ response variables, as follows (Geladi and Kowalski, 1986):

$$
\begin{aligned}
& \mathbf{X}=\mathbf{T} \mathbf{P}^{T}+\mathbf{E} \\
& \mathbf{Y}=\hat{\mathbf{U}} \mathbf{Q}^{T}+\mathbf{F}
\end{aligned}
$$

where $\mathbf{T}$ and $\hat{\mathbf{U}}$ are score matrices, $\mathbf{P}$ and $\mathbf{Q}$ are loading matrices and $\mathbf{E}$ and $\mathbf{F}$ are residual matrices. Note that the predictor matrix $\mathbf{X}$ includes a set of variables that predicts the variation encapsulated in the response matrix $\mathbf{Y}$.

The above decomposition generates an "inner model" and an "outer model". The inner model describes the relationship between the $\mathbf{u}$-score matrix $\mathbf{U}$ and the $\mathrm{t}$ score matrix T. In contrast, the outer model shows the relationship between the predictor and response matrices. The inner and outer models are defined in Equations (3) and (4), respectively:

$$
\begin{aligned}
& \mathbf{U}=\hat{\mathbf{U}}+\mathbf{G}=\mathbf{T B}+\mathbf{G} \\
& \mathbf{Y}=\hat{\mathbf{Y}}+\mathbf{F}=\mathbf{X C}+\mathbf{F}
\end{aligned}
$$

where $\mathbf{G}$ is a residual matrix of the inner model, $\mathbf{B}$ is a diagonal regression matrix, $\hat{\mathbf{Y}}$ is the prediction of $\mathbf{Y}$ and $\mathbf{C}$ is a regression matrix.

In order to update the current PLS model at sample instant $k$, when a new sample $\left\{\mathbf{x}_{k+1}, \mathbf{y}_{k+1}\right\}$ becomes available, PLS regression can be performed with $\left[\begin{array}{c}\mathbf{P}_{k}^{T} \\ \mathbf{x}_{k+1}^{T}\end{array}\right],\left[\begin{array}{c}\mathbf{B}_{k} \mathbf{Q}_{k}^{T} \\ \mathbf{y}_{k+1}^{T}\end{array}\right]$ instead of using the original plant data $\left[\begin{array}{c}\mathbf{X}_{k} \\ \mathbf{x}_{k+1}^{T}\end{array}\right],\left[\begin{array}{c}\mathbf{Y}_{k} \\ \mathbf{y}_{k+1}^{T}\end{array}\right]$ since these produce the same inner and outer regression model (Qin, 1998). This allows a computationally more efficient adaptation technique that is applicable to large-scale processes (Qin, 1998).

\subsection{Monitoring statistics for PCA/PLS related approaches}

The monitoring of industrial processes usually relies on univariate statistics such as (Hotelling's) $\mathrm{T}^{2}$ and the squared prediction error (SPE) (Wise and Gallagher, 1996; Martin and Morris, 1996). Li et al. (2000) and Wang et al. (2003) showed how a recursive version of these monitoring statistics, and their confidence limits, may be obtained for PCA and PLS. Note that PLS produces two SPE statistics, i.e. one residual statistic for the predictor variables $\left(S P E^{(X)}\right)$ and one residual statistic for the response variables $\left(S P E^{(Y)}\right)$. Further, the model obtained at the previous sample instant should be used in calculating the current statistics. This is essential for increasing the sensitivity of the monitoring model in detecting abnormal process behaviour. 
The contributions to individual plant variables to the statistics can be obtained as discussed in Russel et al. (2000) using contribution charts. These enable the variables which contribute most to abnormal plant behaviour to be identified. Our new fault diagnosis charts are similar but incorporate changes in the model coefficients resulting from the recursive adaptation of the PCA/PLS model.

\section{NEW FAULT DIAGNOSIS CHARTS}

This section introduces two new charts that allow changes in the loading matrix (recursive PCA) and the outer regression matrix (recursive PLS) to be displayed. They are therefore tailored for use with recursive versions of PCA and PLS. The effectiveness of these new charts is demonstrated in a simulated application study of a complex chemical process. The derivations of both charts are given below.

\subsection{Changes in the loading matrix for RPCA}

The loading matrix $\mathbf{R}_{k}$ contains the information about how the variables relate to each other at time instant $k$. The elements of this matrix clearly change in value if the PCA model is updated and the process behaviour changes. Given that $\mathbf{R}_{k}$ is an $n$ by $m$ matrix, with $n=M+N$ recorded process variables and $m$ the number of retained principal components, the $i^{\text {th }}$ column of $\mathbf{R}_{k}$ explains how the $i^{\text {th }}$ principal component contributes to the original $n$ process variables.

In order to determine the impact of a particular process variable on the remaining ones, the projection matrix $\mathbf{D}_{k}=\mathbf{R}_{k} \mathbf{R}_{k}^{T}$ is applied here rather than using $\mathbf{R}_{k}$. The latter only determines the impact of a particular principal component on the process variables. The resultant updated PCA model allows following matrix be constructed to,

$$
\Delta \mathbf{D}_{k+1}=\mathbf{D}_{k+1}-\mathbf{D}_{k}
$$

The elements of $\Delta \mathbf{D}_{k+1}$ can be displayed in a 3dimensional bar chart, where the first and second axis refers to individual process variables and the third axis relates to the corresponding element in $\Delta \mathbf{D}_{k}$. Since $\Delta \mathbf{D}_{k+1}$ is symmetric, it is sufficient to plot the upper or lower triangular elements together with the diagonal elements. This allows an easier interpretation of the plotted results.

\subsection{Changes in the regression matrix for RPLS}

Under the assumption that the process is timevarying, the elements in the coefficient matrix $\mathbf{C}$, defined in Equation 4, change when the PLS model is updated. Such changes can be used to provide information on how the relationships between the predictor and response variables vary over time. For in- stance, if the $i^{\text {th }}$ predictor variable encounters a severe change at sampling point $k+1$, elements in the $i^{\text {th }}$ row of $\mathbf{C}_{k+1}$ differ significantly from those in $\mathbf{C}_{k}$ as a result of model adaptation. By comparing the difference between $\mathbf{C}_{k+1}$ and $\mathbf{C}_{k}$, i.e.:

$$
\Delta \mathbf{C}_{k+1}=\mathbf{C}_{k+1}-\mathbf{C}_{k}
$$

The changes in the $i^{\text {th }}$ row can be detected and used to trace this abnormal event back to the $i^{\text {th }}$ predictor variable.

Similarly, the change of a particular column in $\Delta \mathbf{C}_{k+1}$ reflects a change in the corresponding response variable. Examination of significant elements in $\Delta \mathbf{C}_{k+1}$ again allows determining potential root causes, responsible for such an abnormal event, to be determined.

The elements of $\Delta \mathbf{C}_{k+1}$ can also be plotted on a 3dimensional bar chart, where one axis corresponds to the predictor variables, one axis relates to the response variables and the third axis is associated with the elements in $\Delta \mathbf{C}_{k+1}$.

\section{APPLICATION STUDY}

This section shows the utility of the two new contribution charts using data that stems from a realistic simulation of a fluid catalytic cracking unit (FCCU). Both charts are contrasted with each other on the basis of the information content provided to process operators. A description of the FCCU is given prior to results from the detection and diagnosis of a simulated fault scenario.

\subsection{Process description}

A fluid catalytic cracking unit is an important economic unit in refining operations. It typically receives several different heavy feedstocks from other refinery units and cracks these streams to produce lighter, more valuable components that are eventually blended into gasoline and other products. The particular Model IV unit described by McFarlane et al. (1993) is illustrated in Figure 1. The principal feed to the unit is gas oil, but heavier diesel and wash oil streams also contribute to the total feed stream. Fresh feed is preheated in a heat exchanger and furnace and then passed to the riser, where it is mixed with hot, regenerated catalyst from the regenerator. Slurry from the main fractionator bottoms is also recycled to the riser. The hot catalyst provides the heat necessary for the endothermic cracking reactions. The gaseous cracked products are passed to the main fractionator for separation. Wet gas at the top of the main fractionator is increased to the pressure of the downstream separation by the wet gas compressor. Separa- 
tion of light components occurs in this downstream separation section.

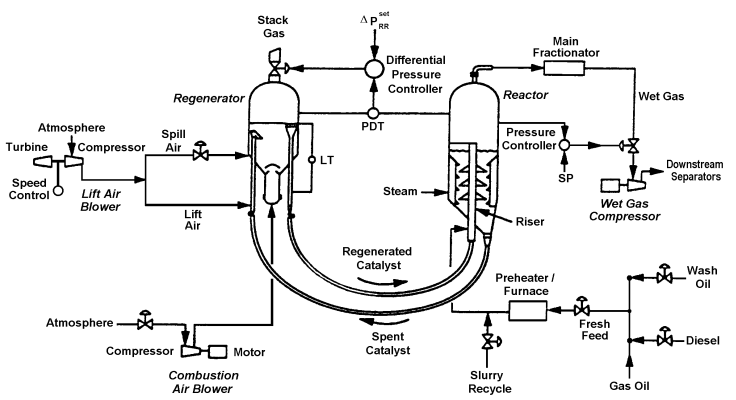

Fig. 1: Schematic diagram of the fluid catalytic cracking unit (McFarlane et al., 1993)

As a result of the cracking process, a carbonaceous material, coke, is deposited on the surface of the catalyst, which depletes its catalytic property. For this reason, spent catalyst is recycled to the regenerator where it is mixed with air in a fluidised bed for regeneration of its catalytic properties. Oxygen reacts with the deposited coke to produce carbon monoxide and carbon dioxide. Air is pumped to the regenerator with a high-capacity combustion air blower and a smaller lift air blower. In addition to contributing to the combustion process, air from the lift air blower assists with catalyst circulation. Complete details of the mechanistic simulation model for this particular model IV FCCU can be found in (McFarlane et al., 1993).

The selected process variables for the FCCU case study are given in Table 1 . In order to produce typical signals that exhibit non-stationary and time varying character, different ARIMA and ARMA signals were superimposed on the predictor variables and the parameters in the simulation.

The set of faulty data under investigation in this paper considers a $1 \%$ degradation in the flow of regenerated catalyst between the regenerator and riser, which is typically caused by partial or complete plugging of steam injectors located in this line (McFarlane et al., 1993). A data set was simulated containing 1150 data points at sampling intervals of 1 minute. The fault in the form of a step, was injected after 950 samples. Physically, any change in regenerated catalyst flow will primarily affect catalyst-tofeed ratio in the riser, resulting in a change in the amount of coke deposited on the spent catalyst and subsequently the level of oxygen usage in the regenerator. Further consequences are related to the material balance in the standpipe, and hence its level, as well as the reaction conditions in the reactor.

\subsection{Fault detection using monitoring charts}

Wang et al. (2003) demonstrated the need for using recursive techniques to monitor this process. Given
Table 1: Considered variables of FCCU Simulator

\begin{tabular}{|c|c|c|}
\hline \multicolumn{2}{|c|}{ Variable No. } & Description \\
\hline \multirow{12}{*}{$\begin{array}{l}\text { Predictor } \\
\text { Variables }\end{array}$} & 1 & Wash oil feed flowrate \\
\hline & 2 & Total fresh feed flowrate \\
\hline & 3 & Slurry flowrate \\
\hline & 4 & Preheater outlet temperature \\
\hline & 5 & Fresh feed temperature to riser \\
\hline & 6 & Furnace firebox temperature \\
\hline & 7 & Comb. air blower suct. flowr. \\
\hline & 8 & Comb. air blower throughput \\
\hline & 9 & Combustion air flowrate \\
\hline & 10 & Lift air blower suct. flowrate \\
\hline & 11 & Lift air blower speed \\
\hline & 12 & Lift air blower throughput \\
\hline \multirow{11}{*}{$\begin{array}{l}\text { Response } \\
\text { Variables }\end{array}$} & 13 & Riser temperature \\
\hline & 14 & Wet gas compr. suct. pressure \\
\hline & 15 & Wet gas compr. suct. flowrate \\
\hline & 16 & Wet gas flowrate to rec. unit \\
\hline & 17 & Regenerator bed temperature \\
\hline & 18 & Reg. stack gas temperature \\
\hline & 19 & Regenerator pressure \\
\hline & 20 & Standpipe catalyst level \\
\hline & 21 & Stack gas $\mathrm{O}_{2}$ concentration \\
\hline & 22 & Comb. blower discharge press. \\
\hline & 23 & Wet gas comp. suct valve pos. \\
\hline
\end{tabular}

that fault diagnosis using recursive MSPC techniques is the focus here, the fault detection results are only described briefly. Figure 2 shows the application of RPLS to this data set. It can be seen that the $S P E^{(Y)}$ statistic from the RPLS approach is giving the strongest response to this event, which is understandable since most of the response variables are affected by the fault.

However, an increase in both the $T^{2}$ and the $S P E^{(X)}$ statistic can also be seen. This is explained by the pressure change in the regenerator as a consequence of the excess production of carbon monoxide and carbon dioxide, which leads to a reduction of the airflow of the combustion as well as the lift air blower. A change in the relationship between the predictor variables is the consequence, which leads to a significantly changed $S P E^{(X)}$ statistic. Since the variation in the air blower variables is excessive, the $T^{2}$ statistic variation is also significant.

The results from applying RPCA are shown in Figure 3 , where both statistics were severely affected by the event at time instant 950. Given the above explanations, these results coincide with those from RPLS.

\subsection{Application of fault diagnosis charts}

The fault was introduced at sampling point 950 . The new fault diagnosis charts were applied to determine which of the variables were most affected by the reduced flow of regenerated catalyst. The matrices, $\Delta \mathbf{D}_{k+1}$ for recursive PCA and $\Delta \mathbf{C}_{k+1}$ for recursive PLS were both obtained for $k=949$. 

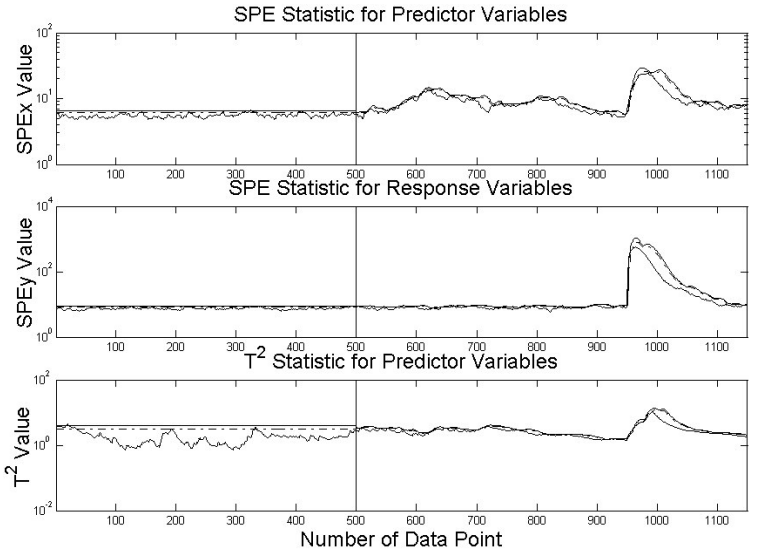

Fig. 2: RPLS monitoring charts FCCU catalyst faulty data
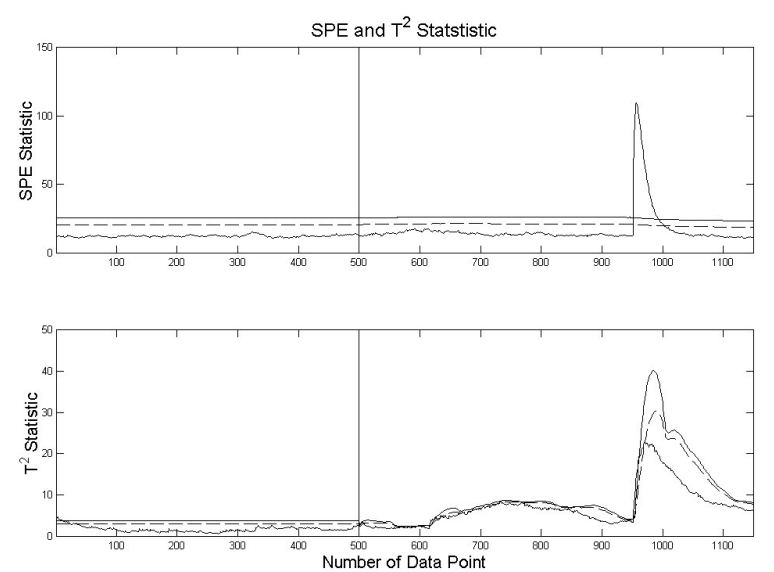

Fig. 3: RPCA monitoring charts on FCCU catalyst faulty data

The elements of $\Delta \mathbf{D}_{k+1}$ at sampling point $k=949$ are plotted in Figure 4. The most affected variables refer to the $20^{\text {th }}$ process variable, which is the standpipe catalyst level. More precisely, the following combinations involving the standpipe catalyst level produced significant changes in $\Delta \mathbf{D}_{950}$ : standpipe catalyst level $\rightarrow$ (i) slurry flowrate, $\rightarrow$ (ii) preheater outlet temperature, $\rightarrow$ (iii) lift air blower suction flowrate, $\rightarrow$ (iv) lift air blower speed and $\rightarrow$ (v) lift air blower throughput. These variables have been correctly identified, as the reaction conditions are affected including the material balance in the standpipe and hence its level. More precisely, the lift air blower assists the catalyst circulation and the feeds, particularly the slurry feed, which all contribute to the reaction. Moreover, the preheater outlet temperature is the temperature with which the input feed enters the reactor. Consequently, this temperature also affects the reaction conditions.

For RPLS, the changes in the regression matrix $\mathbf{C}_{k}$ for $k=949$, i.e. $\Delta \mathbf{C}_{950}$, are shown in Figure 5. The most dominant contributions are noticeable for the $8^{\text {th }}$ response variable, i.e. the standpipe catalyst level. In addition, the same variable pairs as those previously seen with $\Delta \mathbf{D}_{950}$ were also significant in $\Delta \mathbf{C}_{950}$.

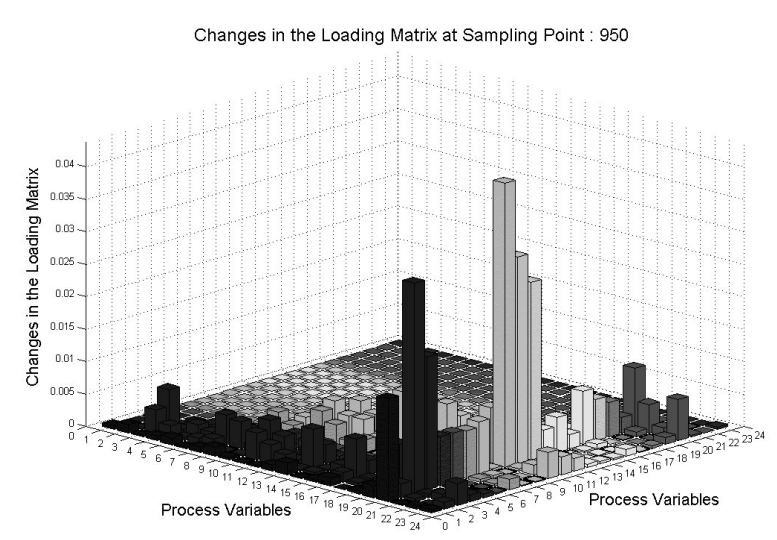

Fig. 4: Changes in matrix D of RPCA model for $k=$ 949

Consequently, both fault diagnosis charts offer the same information to the process operator. The next subsection compares the number of elements in the matrices $\Delta \mathbf{D}_{k+1}$ and $\Delta \mathbf{C}_{k+1}$.

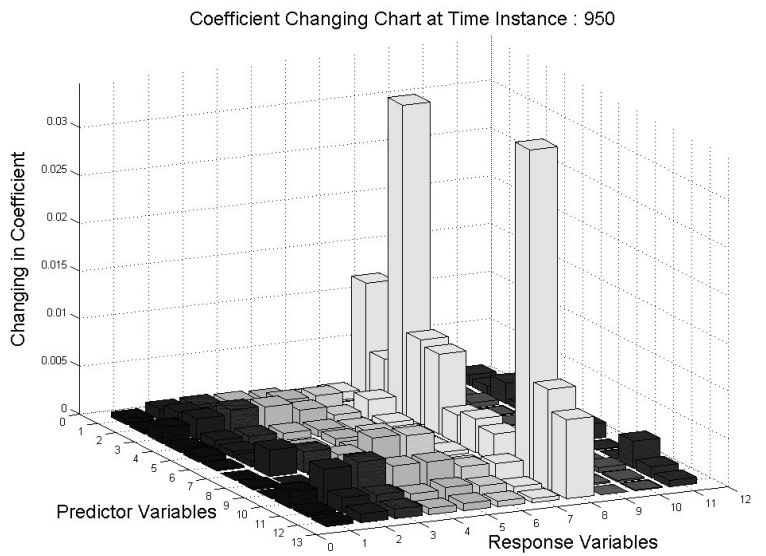

Fig. 5: Coefficient changes in RPLS model for $k=$ 949.

4.4 Number of elements in $\Delta \boldsymbol{D}_{k+1}$ and $\Delta \boldsymbol{C}_{k+1}$

The total number of elements $\Delta \mathbf{D}_{k+1}$ is equal to $(N+$ $M)^{2}$. Given that only the upper or lower triangular elements along with the diagonal elements are required, the number of elements that are displayed, $p_{\mathrm{PCA}}$, in general is given by:

$$
p_{\mathrm{PCA}}=\frac{(N+M+1)(N+M)}{2}
$$

In contrast, the total number of elements in $\Delta \mathbf{C}_{k+1}$, $p_{\text {PLS }}$, is:

$$
p_{\mathrm{PLS}}=N M
$$

Comparing these, it is apparent that in general the regression matrix for recursive PLS includes fewer parameters than in the projection matrix for recursive PLS when: 


$$
M^{2}+N^{2}>M+N
$$

Consequently, the elements displayed in $\Delta \mathbf{D}_{k+1}$ contain redundancy if $M$ or $N>1$, although both matrices produced the same information regarding the fault in the regenerated catalyst. This analysis provides further evidence that, recursive PLS is probably to be preferred to recursive PCA for detecting and diagnosing abnormal process behaviour.

\section{CONCLUSIONS}

This paper introduced two new fault diagnosis charts that are tailored to recursive PCA and PLS based condition monitoring. These display changes in the projection matrix for PCA and the regression matrix for PLS, resulting from the updating of monitoring models.

Application to a realistic simulation of a fluid catalytic cracking unit, where a fault in the regenerated catalyst line was simulated, showed that this event was correctly diagnosed. More precisely, the variable pairs that were mostly affected by this event were identified and would have assisted an experienced operator in narrowing down potential root causes of this event.

A general analysis pf the number of parameters that both techniques display revealed that recursive PLS is to be preferred over recursive PCA.

\section{REFERENCES}

Dayal, B.S. and J.F. MacGregor (1997). Recursive exponentially weighted PLS and its applications to adaptive control and prediction. Journal of Process Control, 7(3), 169-179.

Gallagher, N.B., B.M. Wise, S.W. Butler, D.D. White and G.G. Barna (1997). Development and benchmarking of multivariate statistical process control tools for a semiconductor etch process: improving robustness through model updating. Proc. ADCHEM 97, Banff, Canada, 78-83.

Helland, K., H.E. Berntsen, O.S. Borgen and H. Martens (1991). Recursive algorithm for partial leastSquares regression. Chemometrics and Intelligent Laboratory Systems, 14, 129-137.

Kresta, J.V., J.F. MacGregor and T.E. Marlin (1991). Multivariate statistical monitoring of process operating performance. The Canadian Journal of Chemical Engineering, 69, 35-47.

Kourti, T. and J.F. MacGregor (1995). Process analysis, monitoring and diagnosis using multi variate methods. Chemometrics and Intelligent Laboratory Systems, 28, 3-21.

Li, W., H. Yue, S. Valle-Cervantes and S.J. Qin (2000). Recursive PCA for adaptive process monitoring. Journal of Process Control, 10(5), 471-486

MacGregor, J.F., T.E. Marlin, J.V. Kresta and B. Skagerberg (1991). Multivariate statistical methods in process analysis and control. AIChE Symposium Proceedings of the $4^{\text {th }}$ International Conference on Chemical Process Control, New York: AIChE Publ. No. P-67, 79-99.

MacGregor, J.F., C. Jaeckle, C. Kiparissides and M. Koutoudi (1994). Process monitoring and diagnosis by mulitblock PLS methods. AIChE Journal, 40(5), 826-838.

MacGregor, J.F. and T. Kourti (1995). Statistical process control of multivariate processes. Control Engineering Practice, 3(3), 403-414.

Martin, E.B. and A.J. Morris (1996). An overview of multivariate statistical process control in continuous and batch process performance monitoring. Transactions of the Institute of Measurement and Control, 18(1), 51-60.

McFarlane, R.C., R.C. Reineman, J.F. Bartee and C. Georgakis (1993). Dynamic simulator for a model IV fluid catalytic cracking unit. Computers in Chemical Engineering, 17(3), 275-300.

Morud, T.E. (1996). Multivariate statistical process control; example from the chemical process industry. Journal of Chemometrics, 10, 669-675.

Qin, S.J. (1993). A Recursive PLS algorithm for system identification. AIChE Annual Meeting, St. Louis, USA.

Qin, S.J. (1998). Recursive PLS algorithms for adaptive data modelling. Computers in Chemical Engineering, 22(4/5), 503-514.

Russel, E.L., L.H. Chiang and R.D. Braatz (2000). Data-Driven Techniques for Fault Detection and Diagnosis in Chemical Processes. Springer, London.

Wang, X., U. Kruger and B. Lennox (2003). Recursive partial least squares algorithms for monitoring complex industrial processes, Control Engineering Practice, 11(6), 613-632.

Wise, B.M. and N.B. Gallagher (1996). The process chemometrics approach to process monitoring and fault detection. Journal of Process Control, 6(6), 329-348. 